\title{
Propofol Induced Anaphylaxis-A Case Report
}

Angela Carmezim Mota ${ }^{1}$, Filipa Pereira ${ }^{1}$, Judite Guimaraes ${ }^{2}$, Esmeralda Neves ${ }^{2}$, Paula Sa ${ }^{1}$, Miguel Paiva ${ }^{1}$, Julio Guimaraes $^{1}$ and Humberto Machado ${ }^{{ }^{*}}$

${ }^{1}$ Serviço de Anestesiologia, Centro Hospitalar do Porto, Largo Professor Abel Salazar, 4099-001 Porto, Portugal

2Serviço de Imunologia, Centro Hospitalar do Porto, Largo Professor Abel Salazar, 4099-001 Porto, Portugal

*Corresponding author: Machado H, Serviço de Anestesiologia, Centro Hospitalar do Porto, Largo Professor Abel Salazar, $4099-001$ Porto, Portugal, Tel: 351 935848475; Email: hjs.machado@gmail.com

Received date: March 12, 2015, Accepted date: April 17, 2015, Published date: April 30, 2015

Copyright: (c) 2015 Mota AC, et al. This is an open-access article distributed under the terms of the Creative Commons Attribution License, which permits unrestricted use, distribution, and reproduction in any medium, provided the original author and source are credited.

\begin{abstract}
Perioperative anaphylaxis is an important cause of anesthesia-related mortality and morbidity. The true incidence is unknown and is most likely under reported, but it is believed to be a rare event. Diagnosis can be challenging, particularly because the cause is difficult to identify as a number of drugs commonly used simultaneously can potentially be the cause and they can also mask its manifestations.

We report a case of propofol-induced anaphylaxis during the induction of anesthesia in a $77 \mathrm{y}$-old woman proposed for an orthopedic procedure. The patient was thoroughly studied with immunoallergologic testing that proved that propofol was the underlying cause of this IgE-mediated anaphylactic event.
\end{abstract}

Keywords: Anaphylaxis; Propofol; Propofol allergy

\section{Introduction}

Throughout the years the practice of anesthesiology has become increasingly safe to the point that serious adverse events that may threaten the patient well-being are now considered to be rare. One such example is anaphylaxis, the most severe of immediate hypersensitivity reactions, which may occur during anesthesia [1].

The operating theatre is a unique clinical environment. Patients are exposed to numerous medications and other non-drug substances over a relatively short period of time. As a consequence, anesthesiologists are more likely than most other physicians to witness and manage allergic reactions [2]. In the literature, the reported incidence of perioperative anaphylaxis varies from 1 in 6,000 to 1 in 20,000 anesthetics $[2,3]$.

Almost all the drugs and substances to which patients are exposed perioperatively have been known to cause allergic reactions [4].

Propofol (2-6-diisopropylphenol) is an alkyl phenol in a lipid vehicle (soybean, oil egg lecithin and glycerol). It is a short-acting anesthetic that is administered for induction and maintenance of general anesthesia. Allergic reactions to propofol are uncommon and account for less than $2 \%$ of all reactions to general anesthetics [5].

We report a case of propofol-induced anaphylaxis with bronchospasm and hypotension after induction of anesthesia. We highlight the importance of the discussion between the anesthesia and immunoallergology team as the cornerstone for the diagnosis of this case.

\section{Case Report}

A 77-y-old woman with a history of hypertension, heart failure (NYHA class II), dyslipidemia, obesity (BMI $37 \mathrm{~kg} / \mathrm{m}^{2}$ ) and osteoarticular pathology, was proposed for a right shoulder arthroplasty in our hospital. She didn't have any documented food or pharmacologic allergies.

The patient reported a respiratory complication during induction of anesthesia two years before when she was proposed to an orthopedic surgery in another hospital. At the time this event resulted in the surgery cancellation. There was no clinical documentation about such event.

At the day of the surgery she was monitored according to ASA standards and pre-medicated with midazolam $2 \mathrm{mg}$ IV. BIS ${ }^{\mathrm{Tax}}$ and TOF were used to monitor the depth of anesthesia and the degree of muscular relaxation, respectively. She was submitted to a general anesthesia using fentanyl $0.2 \mathrm{mg}$ IV, propofol $140 \mathrm{mg}$ IV and rocuronium $40 \mathrm{mg} I V$. An endotracheal intubation was performed and a number 7 endotracheal tube was used. Maintenance of anesthesia was accomplished with sevoflurane.

Immediately after endotracheal intubation, about two minutes after propofol administration, a ventilation difficulty was detected with a rise in peak airway pressures, bronchospasm, desaturation with a fall in oxygen peripheral saturation $\left(\mathrm{SpO}_{2}\right)$ and hypotension. There were no signs of cutaneous rash or edema. This clinical situation, not at first recognized as an allergic reaction, was first interpreted as an event related to the induction of anesthesia and was symptomatically treated with a sympathomimetic (total of $40 \mathrm{mg}$ IV of ephedrine), nebulized bronchodilators (salbutamol and ipatropium bromide) and corticosteroids (hydrocortisone $200 \mathrm{mg}$ IV). After the immediate resolution of the problem, and after reviewing the events, the anesthetic team thought this could have been an anaphylactic event. However, measurement of serum tryptase levels was not performed.

The antibiotic prophylaxis with cefazoline $2 \mathrm{~g}$ IV was administered after the resolution of the event. Rocuronium was not administered again after the induction of anesthesia and reversal of neuromuscular blockade was not performed, according to the TOF monitoring. Paracetamol $1 \mathrm{~g} \mathrm{IV}$, parecoxibe $40 \mathrm{mg}$ IV and tramadol $100 \mathrm{mg}$ IV were used for post-operative analgesia. No other events were detected 
Page 2 of 4

during the intraoperative period and no complications occurred during the emergence of anesthesia and extubation.

At the end of the surgery the patient was transferred to the postrecovery unit. No respiratory or hemodynamic complications were reported during the rest of the perioperative period.

The adverse event was carefully reported and explained to the patient. Because an anaphylactic event was suspected she was referred to an immunoallergologic appointment for further evaluation.

After a one year follow-up she was diagnosed with a mild obstructive respiratory disease and medicated with a bronchodilator to be used only in case of symptoms (in SOS).

Two years later she was proposed to a revision of her right shoulder arthroplasty in our hospital. Given the patient's history, she was referred to an anesthetic appointment for preoperative evaluation where she was again referred to the immunoallergologic department before proceeding with the surgery. In this evaluation they discovered that in the past two years she didn't have any bronchospasm event nor she used the SOS bronchodilator, and once again the hypothesis of a previous anaphylactic event was called into question. The patient was then submitted to an extended immunollergologic study.

\section{Methods}

Total IgE serum levels and specific IgE antibodies were determined by a fluorescence enzyme immunoassay - ImmunoCAP ${ }^{\circ} 100$; ThermoFisher, Sweden.

The evaluation of in vitro basophil activation upon antigen stimulation was measured by flow cytometry in a Coulter ${ }^{\circledR}$ Epics XL$\mathrm{MCL}^{\mathrm{rt}}$ Flow Cytometer. The increase of the CD63 (gp53) expression at the cellular surface was measured in EDTA whole blood, using the FLOW CAST $^{\circ}$ Basophil Activation Test (BAT) kit - BUHLMANN, Switzerland, according to the manufacturer's instructions.
The basophil population was gated by the expression of the human chemokine receptor CCR3 labeled with phycoerythrin (anti-CCR3$\mathrm{PE}$ ), constitutively expressed on eosinophils and basophils and low Side Scatter SSC low. The expression of CD63 labeled with fluorescein isothiocyanate (anti-CD63-FITC) was analysed on this gated cell population and the acquisition was performed on 1000 cells for each sample.

A highly specific monoclonal antibody binding to the high affinity IgE binding receptor (FceRI) and the unspecific cell activator fMLP were used as positive controls, the Stimulation Buffer as background negative control and propofol as allergen, in the same conditions, at a dilution of $1 / 40$ and $1 / 160$.

Results are given as the percentage of basophil expressing CD63 and the ratio between this percentage on stimulated and non-stimulated cells. Samples with a raise of more than 5\% of CD63 expression and a stimulation ratio $>2$ were regarded as positive.

\section{Results}

The study revealed an elevated total serum IgE level $(642 \mathrm{kU} / \mathrm{L})$. The patient was studied for several components of the propofol formulation and was negative for soybeans, soy products and egg yolk. However, there was a high value for the egg white which is in its superior limit $(0.37 \mathrm{kUA} / \mathrm{L})$. The patient was also tested for latex allergy with a negative result.

The functional cellular study using the expression of CD63 detected an increase in basophils degranulation after stimulation with propofol (1/160), with a stimulation index of 2.0 (Figures 1 and 2). Using the same concentration (1/160), there was no increase in basophils degranulation after stimulation with fentanyl (stimulation index of 1), rocuronium (stimulation index of 1.2), cefazoline (stimulation index of 1.6) or midazolam (stimulation index of 0.5).
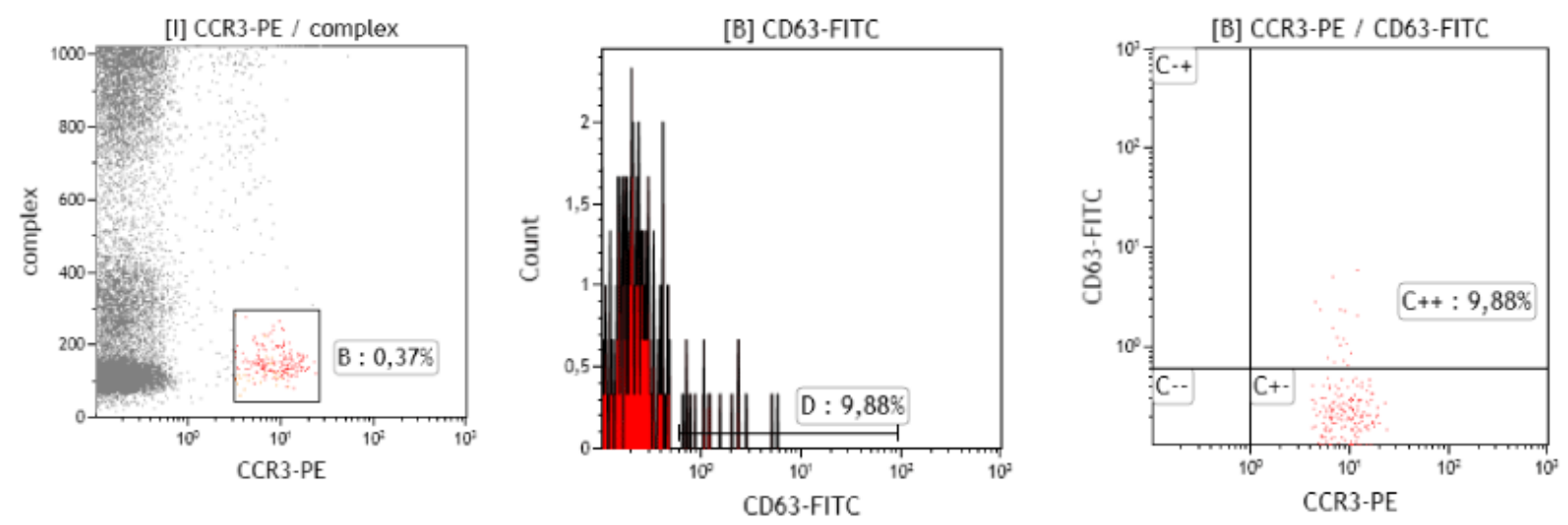

Figure 1: CD63 expression on non-stimulated Basophils 

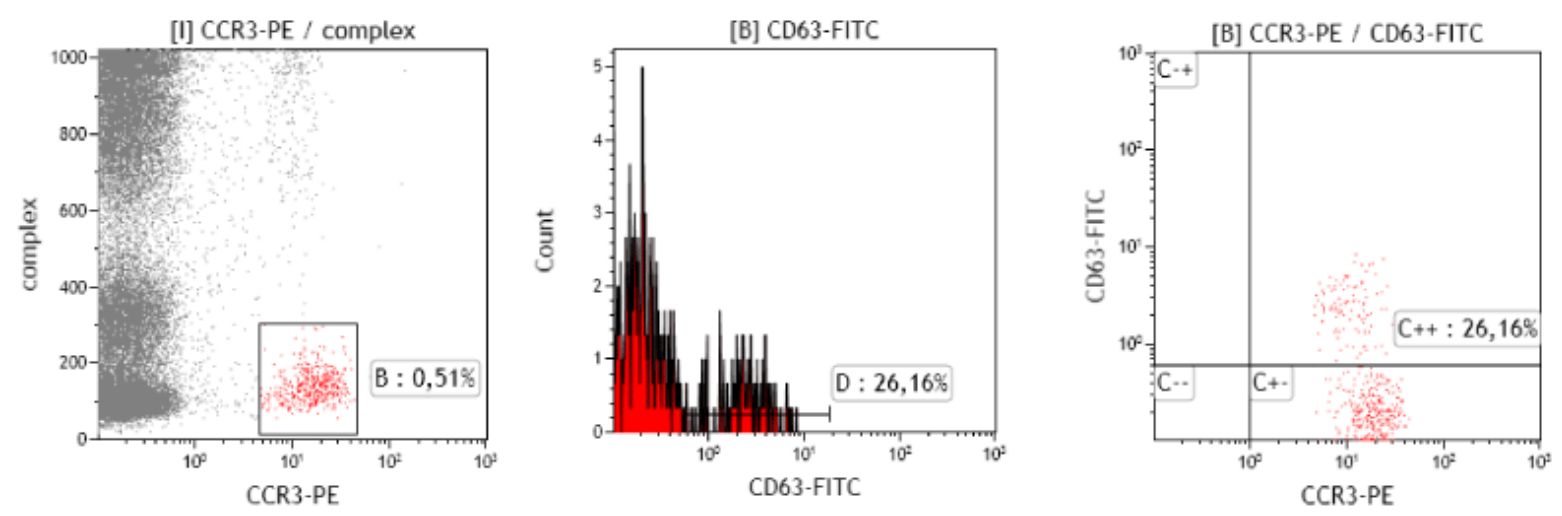

Figure 2: CD63 expression on Basophils stimulated with propofol

After this investigation the patient was submitted to a revision of the right shoulder arthroplasty. Propofol was not used during anesthesia and no complications were reported during the perioperative period.

The patient continued her follow-up in the immunoallergology department and was informed and advised about the cause of her anaphylaxis to prevent future events.

\section{Discussion}

Serious allergic events occurring during anesthesia and the perioperative period are rare, but can rapidly evolve into lifethreatening situations if not rapidly and promptly recognized and managed. Clinical signs are often hard to recognize because they may be masked by the effect of the administered drugs and pathologies of the patient, as happened in this case.

Documentation of anaphylaxis often lacks because the cause and effect relationship is hard to prove and only a minority of patients get referred for allergy testing to confirm the offending drug [6]. In the presented case, based on her previous history and the reported intraoperative event, the patient was referred to the immunoallergology department for further evaluation due to the suspicion of an immunomediated cause. In fact, any unexplained lifethreatening reaction during a previous anesthesia can be an allergic reaction and is a major risk factor for a renewed reaction if the responsible drug is re-administered [7]. So, ideally, all patients having experienced an episode of perioperative anaphylaxis should undergo a complete allergoanesthetic follow-up before further anesthesia.

The study conducted identified propofol as the cause of this anaphylactic event. Rare cases of anaphylaxis following propofol administration have been reported in the medical literature. Although there have been reports of immunoglobulin (Ig) E-mediated reactions (generally urticaria, but sometimes even anaphylactic reactions) [5], non-IgE mediated reactions are more common. Allergic reactions on first exposure are usually because of the isopropyl groups that may act as epitopes and that are present in various medications and cosmetics [8]. Allergic reactions upon re-exposure are usually because of the phenol molecule [7]. Although propofol is not contraindicated in patients who are allergic to egg or soya, some reactions have been associated with the presence of soybean oil or egg lecithin in the solvent of certain commercial propofol preparations [9]. The results obtained when the patient was tested for the egg white can indicate that, although the patient did not have clinical criteria for the diagnosis of egg allergy, she is sensitized to the egg white and this fact should not be neglected given the severity of the clinical presentation and her allergy background.

Regarding the high baseline level of total serum IgE found, since the test was done long after the event of anaphylaxis, this is probably linked to an allergic rhinitis that the patient referred to have when she was younger although no clinical symptoms were described by the patient in recent years.

All the evaluation and study were conducted according to the most recent knowledge, however there was still room for improvement as serum tryptase levels were not measured and first immunoallergologic evaluation was confounded by a mild respiratory disease. Also, skin prick tests and intradermal tests were not performed due to the serious manifestations of this anaphylaxis case and due to the lack of resources to monitor and watch the patient during the performance of these tests.

In conclusion, it is paramount for the anesthesiologist to be prepared to manage the acute event related to an anaphylaxis in the operative room environment, but also to initiate all the immunoallergologic study at an early stage. This case serves as a reminder of that.

In fact, the effective anticipation, prevention, and treatment of these reactions are largely based on the knowledge and vigilance of the attending clinicians and on subsequent allergologic investigations to identify the offending agent and prevent recurrence, since no preemptive therapeutic strategies exist [10]. The anesthesiologist often plays a major role in the identification, treatment and orientation of these cases.

The teamwork between the anesthesiologist and the immunologist is essential for the definitive diagnosis, advice and orientation of future anesthetic drug administration.

\section{References}

1. Mertes PM, Tajima K, Regnier-Kimmoun MA, Lambert M, Iohom G, et al. (2010) Perioperative anaphylaxis. Med Clin North Am 94: 761-789, xi. 
Citation: $\quad$ Mota AC, Pereira F, Guimaraes J, Neves E, Sa P, et al. (2015) Propofol Induced Anaphylaxis-A Case Report. J Allergy Ther 6: 209.

Page 4 of 4

2. Nel L, Eren E (2011) Peri-operative anaphylaxis. Br J Clin Pharmacol 71: 647-658.

3. Saager L, Turan A, Egan C, Mascha EJ, Kurz A, et al. (2015) Incidence of intraoperative hypersensitivity reactions: a registry analysis: a registry analysis. Anesthesiology 122: 551-559.

4. Domínguez-Ortega J, Rodríguez-Jiménez B, González-Herrada C, ElBouayadi L, Kindelan C, et al. (2010) Fixed drug eruption due to propofol after an intradermal test. J Investig Allergol Clin Immunol 20: 629.

5. Dewachter P, Mouton-Faivre C, Castells MC, Hepner DL (2011) Anesthesia in the patient with multiple drug allergies: are all allergies the same? Curr Opin Anaesthesiol 24: 320-325.

6. Mertes PM, Laxenaire MC, Alla F; Groupe d'Etudes des Réactions Anaphylactoïdes Peranesthésiques (2003) Anaphylactic and anaphylactoid reactions occurring during anesthesia in France in 1999-2000. Anesthesiology 99: 536-545.

7. Laxenaire MC, Mata-Bermejo E, Moneret-Vautrin DA, Gueant JL (1992) Life-threatening anaphylactoid reactions to propofol (Diprivan) Anesthesiology 77: 275-280.

8. de Leon-Casasola OA, Weiss A, Lema MJ (1992) Anaphylaxis due to propofol. Anesthesiology 77: 384-386.

9. Hofer KN, McCarthy MW, Buck ML, Hendrick AE (2003) Possible anaphylaxis after propofol in a child with food allergy. Ann Pharmacother 37: 398-401.

10. Mertes PM, Demoly P, Malinovsky JM (2012) Hypersensitivity reactions in the anesthesia setting/allergic reactions to anesthetics. Curr Opin Allergy Clin Immunol 12: 361-368. 